\title{
Risk of Breast Cancer among Young Women and Importance of Early Screening
}

\author{
Zahid Ali Memon ${ }^{1}$, Noureen Kanwal ${ }^{2 *}$, Munam Sami², Parsa Azam Larik², \\ Mohammad Zain Farooq ${ }^{2}$
}

\begin{abstract}
Background: Breast cancer is the most common type of cancer in women throughout the world. However, in comparison with Western women, it presents relatively early in women of Asian ethnicity. Early menarche, late menopause, use of OCP's, family history of benign or malignant breast disease, exposure to radiation and BMI in the under-weight range are well known risk factors for the development of breast cancer in premenopausal women. Early detection with the use of breast self-examination (BSE) and breast cancer screening programs can lead to a reduction in the mortality rates due to breast cancer. The aim of our study was to assess the risk factors for breast cancer among young women and to emphasize the importance of early screening among them. Materials and Methods: We conducted a cross-sectional study among women aged 18 to 25 using a selfadministered questionnaire. Data was collected over a period of 6 months from June to December, 2014. A total of 300 young women selected randomly from Dow Medical College and various departments of Karachi University successfully completed the survey. Results: Respondents were 18-25 years of age (mean age=21.5). Out of the 300 young females, $90(30 \%)$ had at least one risk factor, $90(30 \%)$ had two, $40(13 \%)$ had three, $8(2.7 \%)$ had four, $2(0.7 \%)$ had five while one female was found to have six positive risk factors for breast cancer. Some 66 women (22\%) experienced symptoms of breast cancer such as non-cyclical pain and lumps. While 222 women (74\%) had never performed breast self-examination, $22(7.3 \%)$ had had a breast examination done by a health professional while $32(10.7 \%)$ had participated in breast screening programs. A total of $223(74.3 \%)$ women considered breast cancer screening important for young women. Conclusions: The percentage of young women with risk factors for breast cancer was found to be alarmingly high. Therefore, screening for breast cancer should start at an early age especially in high risk groups. Awareness about breast self-examination should be emphasized. Moreover, screening programs should be started to ensure early detection and reduction of mortality rates caused by breast cancer also in young Pakistani females.
\end{abstract}

Keywords: Breast cancer screening - breast self-examination - young women - risk - Pakistan

Asian Pac J Cancer Prev, 16 (17), 7485-7489

\section{Introduction}

Breast cancer is the most common type of cancer in women in Pakistan and worldwide. However, the mean age at presentation in Pakistan is younger as compared to the Western countries. According to a survey conducted in Karachi, Pakistan, between years 1995 and 1997, the average incidence of Breast Cancer presentation between ages 15 to 24 was $2.9 \%$ and between 25 to 37 years was as high as $27.4 \%$ which was the greatest as compared to any other Cancer presenting at these age groups. This makes Breast Cancer the earliest presenting of all the cancers in women and age standardized rate (ASR) of 515 which is the highest for any Asian country (Bhurgri et al., 2000). At the time of presentation, most cases are of larger size and already show nodal metastasis. A study revealed that according to Modified Bloom and Richardson system of grading, $65 \%$ were Grade II and $24 \%$ were Grade III lesions while $70 \%$ were greater than $2 \mathrm{~cm}$ in size and showed metastasis to greater than 3 lymph nodes at the time of presentation (Siddiqui et al., 2000).

Premenopausal early presenting breast cancer has worse prognosis as compared to late onset (Maureen et al., 1996). It has a higher recovery rate if diagnosed at an early age. It is not yet established why the rates of premenopausal breast cancer in Pakistan are so high (Foti et al., 2005). However, studies suggest that in developing countries, it is largely because of the delay in seeking medical attention (Awadelkarim et al., 2008). Therefore, it is important to carry out screening programs among asymptomatic women so that the tumour can be diagnosed early. Screening should not be restricted to old age (Foti et al., 2005).

Clinical breast examination along with breast selfexam (BSE) can lead to early detection of breast cancer and can be used as an essential screening tool (David et

${ }^{I}$ Surgical Unit 1, Civil Hospital and Dow Medical College, ${ }^{2}$ Final Year S tudent, MBBS, Dow Medical College, DUHS, Karachi, Pakistan *For correspondence: kanwalnoureen@gmail.com 
al., 2002; Noel S. Weiss, 2003). American Cancer Society recommends that women in 20 s and 30 s should get a clinical breast exam done by a health professional every 3 years (American Cancer Society 2015). Early menarche, late menopause, repeated exposure to radiations, family history of benign/ malignant breast disease, cigarette smoking, use of OCP's are the well-established risk factors for Breast Cancer (Bailey and Love's Short Practice of Surgery, $26^{\text {th }}$ edition).

The purpose of our study was to assess the groups among young girls with one or more risk factors or symptoms of Breast Cancer and to assess whether they have ever been screened for the disease. We also intended to assess the knowledge and awareness of Breast Cancer screening in young women.

\section{Materials and Methods}

It was a cross-sectional descriptive study conducted in Dow Medical College and Karachi University over a period of 6 months from 1st June, 2014 to 30th December, 2014.300 questionnaires were filled. Out of 300 questionnaires, 150 were filled from undergraduate female students of Dow Medical College and 150 from undergraduate female students of Karachi University. Data was collected and analyzed on SPSS version 16 .

The questionnaire is comprised of questions regarding various risk factors for breast cancer. Questions regarding marital status, Body Mass Index, age of menarche, repeated exposure to radiations (chest $\mathrm{X}$ ray etc.), family history of any benign or malignant breast disease, breast lump, breast tenderness, age of menopause, menstrual cycle regularity exercise, and use of OCP's were included. Also the subjects were asked about breast examination by themselves or by a health professional or use of any modality for screening were also included .In the end, it also included question to analyze the importance of breast cancer screening among young females.

The study included young females between the age group 18 to 25 . They were explained before filling the questionnaires that the information collected was for research purposes and informed consent was taken. Questionnaires were filled by the subjects voluntarily. The subjects were told that the information will be kept confidential. The study was approved by ethical committee of Dow Medical College.

\section{Limitations of our study}

i) Data was only collected from females who were undergraduates between the age group of 18 to 25. ii) Being a cross-sectional study, data was collected from questionnaires filled by the eligible subject themselves. Reliance was on the person who answered those questions. iii) Data was collected from limited educational institutions.

\section{Results}

A total of 300 women participated in the study. Respondents were 18-25 years of age (Mean age=21.5). Twenty nine $(9.7 \%)$ respondents were married.
We assessed risk factors for the development of female breast cancer. A total of 10 (3.3\%) females had early menarche, $15(5 \%)$ females had positive history of oral contraceptive (OCP's) use, 34 (11.3\%) had been repeatedly exposed to radiations and $92(30.7 \%)$ had family history of late menopause. 31 (10.3\%) women were underweight which places them in a risk group for developing breast cancer. Furthermore, 45 (15\%) women also had positive family history for breast cancer. Participants' risk factors of breast cancer found in our study are summarized in Figure 1.

We also assessed factors which protect against female breast cancer development and it was found that a total of $40(13.3 \%)$ females had irregular menstrual cycles and 74 $(24.7 \%)$ females used to perform regular exercise.

We also cumulated various risk factors present in each individual respondent. It was found that a total of $90(30 \%)$ females had only one risk factor, another 90 females $(30 \%)$ had two and $40(13 \%)$ females had three risk factors for breast cancer. $8(2.7 \%)$ females were found to have four risk factors while $2(0.7 \%)$ were having five positive risk factors for breast cancer. Only one female was found to be having six positive risk factors for breast cancer development. Sum of various risk factors found in individual respondents are shown in Figure 2.

Attitude and practice towards breast cancer of those individuals who were found to be having positive risk factors for breast cancer development were also assessed.

Among those who had BMI in the underweight range $(n=31), 19(61.3 \%)$ had never performed breast selfexamination, $26(83.9 \%)$ did not go to a doctor for breast examination, $22(71 \%)$ did not participate in any breast cancer screening program and $5(16.1 \%)$ said that breast cancer screening in young females is not important

Among those who had positive family history of breast cancer ( $n=45), 29(64.4 \%)$ had never performed self-breast examination, $37(82.2 \%)$ did not go to a doctor for breast examination, $37(82.2 \%)$ did not participate in any breast cancer screening program and $5(11.1 \%)$ said that breast

Risk Factors for female breast cancer development

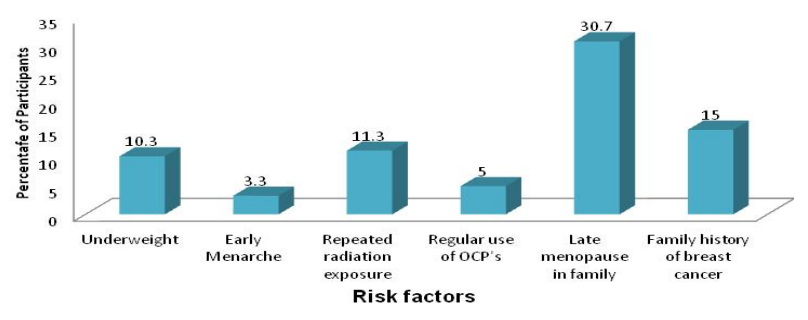

Figure 1. Percentage of Participants Having Risk Factors for Developing Breast Cancer

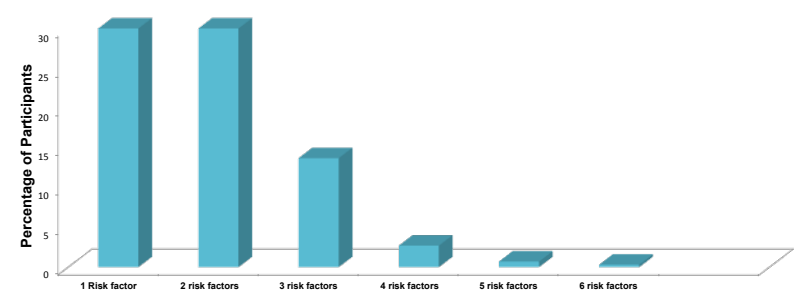

Figure 2. Sum of Risk Factors Found in Individual Respondents 


\begin{tabular}{|c|c|c|c|c|c|}
\hline $\begin{array}{c}\text { Risk factors for Breast Cancer } \\
\text { Development }\end{array}$ & $\mathrm{n}$ & $\begin{array}{c}\text { Never } \\
\text { performed BSE } \\
(\%)\end{array}$ & $\begin{array}{l}\text { Did not go to a } \\
\text { doctor for breast } \\
\text { examination } \\
(\%)\end{array}$ & $\begin{array}{c}\text { Did not participate } \\
\text { in any Breast } \\
\text { cancer Screening } \\
\text { Program } \\
(\%)\end{array}$ & $\begin{array}{c}\text { According to } \\
\text { whom breast cancer } \\
\text { screening in young } \\
\text { females is not } \\
\text { important } \\
(\%)\end{array}$ \\
\hline Family history of Breast Cancer & 45 & 64.4 & 82.2 & 82.2 & 11.1 \\
\hline Early menarche & 10 & 90 & 90 & 100 & 20 \\
\hline Low BMI & 31 & 61.3 & 83.9 & 71 & 16.1 \\
\hline Repeated exposure to radiations & 34 & 73.5 & 79.4 & 82.4 & 5.9 \\
\hline Use of OCP's & 15 & 33.3 & 93.3 & 93.3 & 26.7 \\
\hline Family history of late menopause & 92 & 77.2 & 92.4 & 91.3 & 13 \\
\hline
\end{tabular}

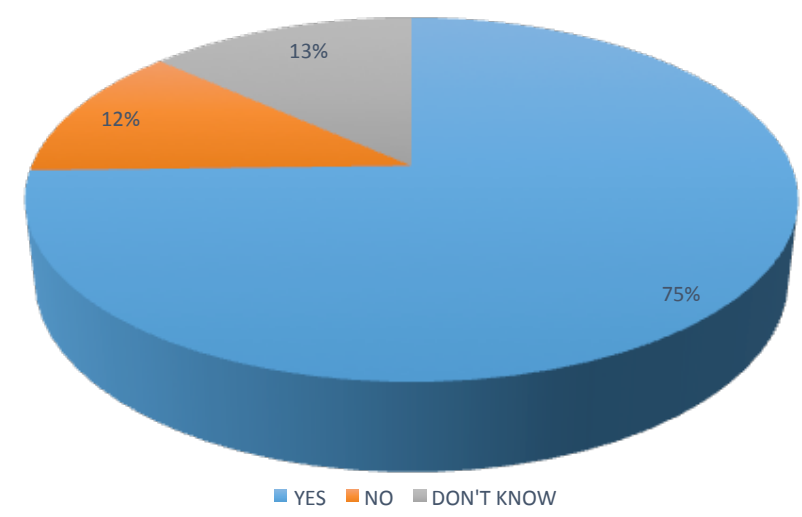

Figure 3. Respondent's Attitude Towards Breast Cancer Screening in Young Females

cancer screening in young females is not important.

Those who had been repeatedly exposed to radiations $(n=34), 25(73.5 \%)$ had never performed self-breast examination, 27 (79.4\%) did not go to a doctor for breast examination, $28(82.4 \%)$ did not participate in any breast cancer screening program and $2(5.9 \%)$ said that breast cancer screening in young females is not important.

Among those who had early menarche $(n=10), 9(90 \%)$ had never performed breast self-examination, $9(90 \%) \mathrm{did}$ not go to a doctor for breast examination, 10(100\%) did not participate in any breast cancer screening program and $2(20 \%)$ considered breast cancer screening in young females unimportant.

Those who had family history of late menopause $(n=92), 71(77.2 \%)$ had never performed breast selfexamination, 85 (92.4\%) did not go to a doctor for breast examination, $84(91.3 \%)$ did not participate in any breast cancer screening program and $12(13 \%)$ said that breast cancer screening in young females is not important.

Among those who had positive history of oral contraceptive (OCP) use $(n=15), 5(33.3 \%)$ had never performed breast self-examination, 14 (93.3\%) did not go to a doctor for breast examination, 14 (93.3\%) did not participate in any breast cancer screening program and 4 $(26.7 \%)$ said that breast cancer screening in young females is not important. (Table no 1)

When asked about symptoms which may be suggestive of breast cancer, 24 (8\%) had lump in breast and 42 (14\%) had history of pain in breast.

We also assessed respondents' attitude and practice towards breast cancer associated symptoms. Among those who had pain or tenderness in breast $(n=42), 28$ $(66.7 \%)$ had never performed BSE, $36(85.7 \%)$ did not go to a doctor for breast examination, $34(81 \%)$ did not participate in any breast cancer screening program and 5 $(11.9 \%)$ said that breast cancer screening in young females is unimportant.

Those who had breast lump $(n=24), 12(50 \%)$ had never performed BSE, $13(54.2 \%)$ never went to a doctor for breast examination, $15(62.5 \%)$ did not participate in any breast cancer screening program and 5 (20.8\%) said that breast cancer screening in young females is not important.

Respondents' practices relative to breast cancer screening and use of any intervention for early detection were also assessed. Majority (74\%) of the females had never performed BSE. Moreover 278 (92.7\%) females never got their breast examination done by a health professional and $268(89.3 \%)$ did not participate in any screening program for breast cancer.

Response regarding the importance of breast cancer screening in young females is shown in figure 3 .

\section{Discussion}

Although the prevalence of breast cancer among young women is extremely low, young age is a bad prognostic factor for the treatment of breast cancer (Maureen et al., 1996; Peter et al., 2002; ShMamoon et al., 2003).Study suggests that young age is the second most powerful risk factor among premenopausal women second only to lymph node status (Peter et al., 2002). Therefore, early detection is important in this age group (ShMamoon et al., 2003). In spite of this vast research-based evidence, assessment of risk factors and importance of screening among younger age group is not much stressed upon.

According to the National Cancer Institute's PDQ cancer information summary about breast cancer, early menarche is defined as beginning of menstrual periods at or before age 11. Early Menarche, exposure to radiation for diseases such as Hodgkin's lymphoma before Age 20 and 
use of OCP's are few of many evidence based risk factors for the development of Breast Cancer. However, vigorous exercise, for 4 or greater than 4 hours a week, significantly reduces risk (Bethesda, 2014). Also, in a study conducted in Netherlands, results showed that women who engaged themselves in any kind of physical activity irrespective of their age had a reduced risk of breast cancer (Janneke et al., 2000).

In our study, $10.3 \%$ women had BMI in underweight range, $3.3 \%$ reported to have had early menarche, $11.4 \%$ said they had been exposed to radiations earlier in life. All these factors put them under a risk of developing breast cancer. However, the percentage of women taking regular exercise was also high (75.3\%) which is protective against breast cancer.

Family history of breast cancer is another established risk factor (Deirdre et al., 2002). A similar study conducted in Turkey revealed that the risk increases 5.7 times in a woman who has a first degree relative suffering from breast cancer (Gokdemir-Yazar et al., 2014). In our study, a total of $15 \%$ women reported to have a positive family history for breast cancer which is slightly less than the percentage observed among Female British University Students which was reported to be $17.2 \%$ (Kanayo et al., 2010).

Result of one study suggested that delay in the age of menarche each year reduces the risk of breast cancer by $6 \%$. Women who had regular periods had an increased risk of breast cancer as compared to those with a an irregular cycle (Wu., et al 1996). In our study, $13 \%$ of the females had irregular cycles, which is protective against breast cancer.

In order to determine whether breast pain is a risk factor for breast cancer, a study was conducted in Ghana, Accra. The results of which showed that breast pain alone is not strongly associated with breast cancer as $63 \%$ of the patients had no significant abnormality and only $1.24 \%$ were found to have breast cancer .Nevertheless, it is stressed that reassurance is not the only solution for females presenting with breast pain. It is recommended to have a breast examination done (Clegg-Lamptey et al., 2007). In our study, $14 \%$ of the females had pain as the only symptom and only $9.5 \%$ went to see a doctor.

Results of one study showed that the lower the BMI, the higher the risk of developing breast cancer in premenopausal women (Margot et al., 1997). in our study, $10.3 \%$ females were underweight and hence they were at increased risk of developing breast cancer.

In a study, it was observed that the median age of menopause in Asian women is 42.1 to 49.5 years while in European women, it is 50.1 to $52.8,50.5$ to 51.4 in North America and is 43.8 to 53 years in women of Latin America (Palacios et al., 2010). Hence, we defined late menopause as Menopause occurring at or after age 50 . The percentage of young women with family history of menopause at age 50 or higher was observed to be as high as $30.7 \%$.

In a study conducted in Yemen, the rate of breast cancer among women presenting with a breast lump was as high as $26.9 \%$, with occurrence at younger age than Western countries (Alsanabaniet al). In our study, 50\% women with a breast lump reported to have consulted a doctor and $37.5 \%$ had ever used a modality for the purpose of screening. This percentage is higher than a study conducted among adolescent girls in Sri Lanka in which only $23.6 \%$ said they would go to a doctor if they ever felt a lump in their Breast (Ranasinghe et al., 2013).

Results of one study conducted in Norway and Sweden showed that use of OCP's ever in life increases breast cancer risk to more than $30 \%$. Moreover, the study also showed that the risk increased to $60 \%$ in recent users while in former users, it increased to $20 \%$ (Kumle et al., 2002). In our study, $5 \%$ females had a positive history of OCPs use which makes them susceptible to breast cancer.

Study suggests that since mammography is not sensitive enough to pick neoplastic changes in younger breast tissue due to increased density, BSE seems to be the only effective method of screening in young women (Kanayo et al., 2010). In our study, only $26 \%$ females reported having performed BSE while the remaining $74 \%$ had never performed one. These results are similar to the ones observed in study conducted to assess the knowledge, attitude and awareness of urban women in Karachi in which $25.9 \%$ women reported regularly performing BSE. In the same study, higher mean screening scores were associated with higher education level, income and employment status (Sobani et al., 2012). Compared with Tehran, Iran where beliefs and behaviors of Iranian teachers towards BSE were observed, only $6 \%$ of them reported to be performing BSE on a regular basis which is lower than the one observed in our study (Jarvandia et al., 2002).

Among Srilankan adolescent girls, only $17.1 \%$ knew how to perform breast self-examination and $6.17 \%$ of them had ever performed one. Students who had a family history of breast cancer were significantly more likely to be aware of BSE compared to those without a family history (Ranasinghe et al., 2013). In another study conducted in young Malaysian women in order to know the practice and barriers towards BSE, 55.4\% were found to have performed one. Of those who never performed BSE, $20.3 \%$ had inadequate knowledge about it, $14.3 \%$ were asymptomatic and $4.4 \%$ of them had a fear that they would be diagnosed with breast cancer (Al-Naggar et al., 2011). In a study conducted in Korea in which the Precaution Adoption Process Model (PAPM) was used, it was documented that among women who had not performed BSE in the last six months, $9.4 \%$ had never heard about BSE. (Choi et al., 2015)

Majority of the participants in our study considered breast cancer screening important for young women. In spite of the awareness, majority of them failed to participate in any sort of screening program. The American Cancer society recommends young women to get their breast examination by a health professional every three years (American Cancer Society 2015). We observed reluctance in seeking medical attention from a health professional by the participants. Study suggests that the delay in seeking medical attention is the reason why the mortality rates of breast cancer are so high in Asia (Awadelkarim et al., 2008). Surveys have shown that it may be attributed to the fact that women don't 
consider themselves at-risk for breast cancer and fail to identify potential symptoms for the disease. Moreover, fear and embarrassment may be contributory (Grunfeld et al., 2002). A study suggested that the most frequent reason for delay in seeking medical attention was due to considering the symptoms as harmless (Memon et al., 2013). There is an increasing need to introduce a tailored message for behavior towards breast cancer screening (Choi et al., 2015).

The results of this study suggests that young females consider breast cancer screening important and it should be started at an early age especially in those with risk factors. However, there is only a small percentage of females with risk factors who had gone for breast selfexamination, or examination by a health professional or used any modality for screening. Therefore, it is highly recommended to design breast cancer screening programs for young premenopausal women. Further studies need to be conducted to find out the most appropriate screening modality for young women. Breast health programs should be organized so that mortality caused by cancer can be reduced.

\section{References}

AH Wu, RG Ziegler, MC Pike, et al (1996). Menstrual and reproductive factors and risk of breast cancer in AsianAmericans. British J Cancer, 73, 680-6

Al-Naggar RA, Al-Naggar DH, Bobryshev YV, et al (2011). Practice and barriers toward breast self-examination among young Malaysian women. Asian Pac J Cancer Prev, 12, 1173-8.

American Cancer Society (2015) Breast cancer prevention and early detection

Awadelkarim KD, Arizzi C, Elamin EO, et al (2008). Pathological, clinical and prognostic characteristics of breast cancer in central sudan versus northern italy: implications for breast cancer in Africa. Histopathol, 52, 445-56.

Bailey and Love's Short Practice of Surgery Vol II, Derek Alderson, Gina M, Jonathan R, et al (2013) CARCINOMA OF THE BREAST. In "The Breast", Norman Williams, Christopher Bulstrode, P Ronan O'Connell. CRC press Taylor \& Francis group, page 808-9

Bethesda (2014). National cancer institute: PDQ ${ }^{\circledR}$ breast cancer prevention. national cancer institute.

Bhurgri Y, Bhurgri A, Hassan, et al (2000), Cancer incidence in karachi, pakistan: first results from karachi cancer registry. Int J Cancer, 85, 325-9.

Clegg-Lamptey JN, Edusa C, Ohene-Oti N, Tagoe JA (2007). Breast cancer risk in patients with breast pain in Accra, Ghana. East Afr Med J, 84, 215-8.

David B. Thomas, Dao Li Gao, Roberta M Ray, et al (2002). Self-randomized trial of breast self-examination in shanghai: final results. JNCI J Natl Cancer Inst, 94, 1445-57.

Deirdre A. Hill, Susan Preston-Martin, Ronald K. Ross, Leslie Bernstein (2002). Medical radiation, family history of cancer, and benign breast disease in relation to breast cancer risk in young women. Cancer Causes and Control, 13, 711-8.

E A Grunfeld, A J Ramirez, M S Hunter, M A Richards (2002). Women's knowledge and beliefs regarding breast cancer. $\mathrm{Br}$ J Cancer, 86, 1373-8.

Foti E, Mancuso S (2005). Early breast cancer detection. Minerva Ginecol, 57, 269-92.

Gokdemir-Yazar O, Yaprak S, Colak M, et al (2014). Family history attributes and risk factors for breast cancer in Turkey.
Asian Pac J Cancer Prev, 15, 2841-6.

Hasanthika M Ranasinghe, Nilakshika Ranasinghe, Chaturaka Rodrigo, Rohini De A Seneviratne, Senaka Rajapakse (2013) Awareness of breast cancer among adolescent girls in Colombo, Sri Lanka: a school based study. BMC Public Health, 13, 1209.

Jamila Ali Alsanabani, Waleed Gilan, Azzan Al Saadi (2015). Incidence data for breast cancer among yemeni female patients with palpable breast lumps. Asian Pac J Cancer Prev, 16, 191-4,

Janneke Verloop, Matti A. Rookus, Karin van der Kooy, Flora E. van Leeuwen (2000). Physical activity and breast cancer risk in women aged 20-54 years. JNCI J Natl Cancer Inst, 92, 128-35.

Jarvandia S, A Montazeria, I Harirchib, A Kazemnejadc (2002). Beliefs and behaviours of Iranian teachers toward early detection of breast cancer and breast self-examination. Public Health, 116, 245-9

Kanayo Umeh, Leonnie Jones (2010). Mutually dependant health beliefs associated with Breast Self-examination in British Female University Students. J Am College Health, 59, 126-31

Margot P. Cleary, Nita J. Maihle (1997). The role of body mass index in the relative risk of developing premenopausal versus postmenopausal breast cancer. Proc Soc Exp Biol Med, 216, 28-43

Maureen Chung, Helena R. Chang, Kirby I. Bland, Harold J. Wanebo (1996), Younger women with breast carcinoma have a poorer prognosis than older women. Cancer, 77, 97-103.

Memon ZA, Shaikh AN, Rizwan S, Sardar MB (2013). Reasons for patient's delay in diagnosis of breast carcinoma in Pakistan. Asian Pac J Cancer Prev, 14, 7409-14.

Merethe Kumle, Elisabete Weiderpass, Tonje Braaten, et al (2002). Use of oral contraceptives and breast cancer risk. the norwegian-swedish women's lifestyle and health cohort study. Cancer Epidemiol Biomar Prev, 11, 1375-81.

Noel S. Weiss (2003). Breast cancer mortality in relation to clinical breast examination and breast self-examination. Breast J, 9, 86-9.

Palacios S, Henderson VW, Siseles N, Tan D, Villaseca P (2010). Age of menopause and impact of climacteric symptoms by geographical region.Climacteric, 13, 419-28.

Peter C. Dubskya, Michael F.X. Gnanta, Susanne Tauchera, et al (2002). Young age as an independent adverse prognostic factor in premenopausal patients with breast cancer. Clinical Breast Cancer. Volume 3 (Issue 1), 65-72

ShMamoon, Smith IE (2003). Breast cancer in adolescents and young women. Eur J Cancer, 39, 2632-42.

Siddiqui MS, Kayani N, Sulaiman S, et al (2000). Breast carcinoma in Pakistani females: a morphological study of 572 breast specimens. J Pak Med Assoc, 50, 174-7.

Sobani ZU, Saeed Z, Baloch HN, et al (2012). Knowledge attitude and practices among urban women of Karachi, Pakistan, regarding breast cancer. J Pak Med Assoc, 62, 1259-64.

Sora Choi, Heeyoung So, Myonghwa Park (2015). Predictors of progress in the stage of adoption of breast cancer screening for Korean women. Asian Pac J Cancer Prev, 16, 2637-43 